\title{
Observation of Non-uniformities in Calcium Aluminosilicate Glass using EELS
}

\author{
Z. Yu ${ }^{*}$ and J. Silcox ${ }^{* *}$ \\ * Physics Department, Cornell University, Ithaca, NY 14853 \\ ** School of Applied and Engineering Physics, Cornell University, Ithaca, NY 14853
}

Figure 1 shows an ADF image of a glass particle that is relatively uniform and homogeneous. Almost every other image of a glass particle is similar to this one and there are no evident intensity variations or other differences that reflect whether the composition of one particle is different from another one. Indeed, given that glass is normally amorphous so that there are no large-scale structural variations this observation is so common that it is taken for granted. However, compositional variations are definitely possible and may have a significant effect on the properties of the material. In this paper we report observations of inhomogeneities in glass by the exploitation of localized EELS spectra.

EELS can be effectively used to analyze the chemical composition of material. A high-resolution STEM equipped with an efficient parallel EELS detector can reveal local electronic structures or local chemical compositions by forming an atomic scale focused probe on the sample and collecting the energy loss spectra from that small region. Calcium silicate glasses have a very extensive composition range and have attracted considerate academic and industrial interest ${ }^{1}$. They are considered to have a continuous random network of corner-shared $\mathrm{SiO} 4$ and $\mathrm{AlO}^{-}$tetrahedrons ${ }^{2}$. As a result, it is conventionally assumed that the distributions of $\mathrm{Si}$ and $\mathrm{Al}$ are homogeneous and uniform. This paper presents the EELS observations of non-uniformity of the distribution of $\mathrm{Si}$ and $\mathrm{Al}$ elements in $42 \mathrm{CaO}-$ $25 \mathrm{Al}_{2} \mathrm{O}_{3}-33 \mathrm{SiO}_{2}$ glass.

The electron microscopy specimens are made by grinding the glass into a suspension of small particles (with sizes from hundred? to microns) in acetone. Some of the particles are suspended onto a holey carbon film covered copper grid. This process took only 5-10 minutes and the individual glass particles are assumed to maintain the original chemical distribution of the bulk (i.e., no phase separation occurred during the process of grinding). The experiments were done on the Cornell VG HB501 100kV UHV STEM. Caution was taken to take EELS spectra from thin and no carbon film regions from various glass particles. To reduce radiation damage, a series of spectra were taken from different spots for each region of interest and averaged to increase signal/noise ratio after subtracting a power-law-like background.

Figure 2 and 3 show the EELS spectra for O K-edge and Al, Si L-edges from two different glass particles. There is a striking difference in the $\mathrm{O}$ K-edge. The strong pre-edge peak at about $529 \mathrm{eV}$ detected from one particle represented by the dashed line in Figure 2 is believed to arise from molecular $\mathrm{O}_{2}$ generated by the electron radiation ${ }^{3}$. However, no such peak can be detected in the $\mathrm{O} \mathrm{K}$ edge from another particle represented by the solid line no matter how long the EELS collection time was. We refer to the former particle as $\mathrm{O}_{2}$-detectable particle and the latter $\mathrm{O}_{2}$-undetectable particle. By taking $\mathrm{O} \mathrm{K}$-edge spectra from many different glass particles, we estimate the ratio of the numbers of the $\mathrm{O}_{2}$-detectable particles to that of the $\mathrm{O}_{2}$-undetectable ones is roughly 9:1. The major difference between these two particles is that the $\mathrm{O}_{2}$-undetectable particle is significantly richer with $\mathrm{Al}$ than the $\mathrm{O}_{2}$-detectable particle does. In fact the $\mathrm{Al} / \mathrm{Si}$ ratio obtained by integrating the corresponding peaks in Figure 3 is about $35 \%$ higher for the $\mathrm{O}_{2}$-undetectable particle. 


\section{References:}

$1 \quad J$. F. Stebbins and Z. Xu, Nature 390 (1997) 60.

2 W. H. Zachariasen, Journal of American Chemical Society 54 (1932) 3841.

3 N. Jiang et al., submitted for publication.

4 This work was supported by the Cornell Center for Materials Research (CCMR), a Materials Research Science and Engineering Center of the National Science Foundation (DMR0079992).
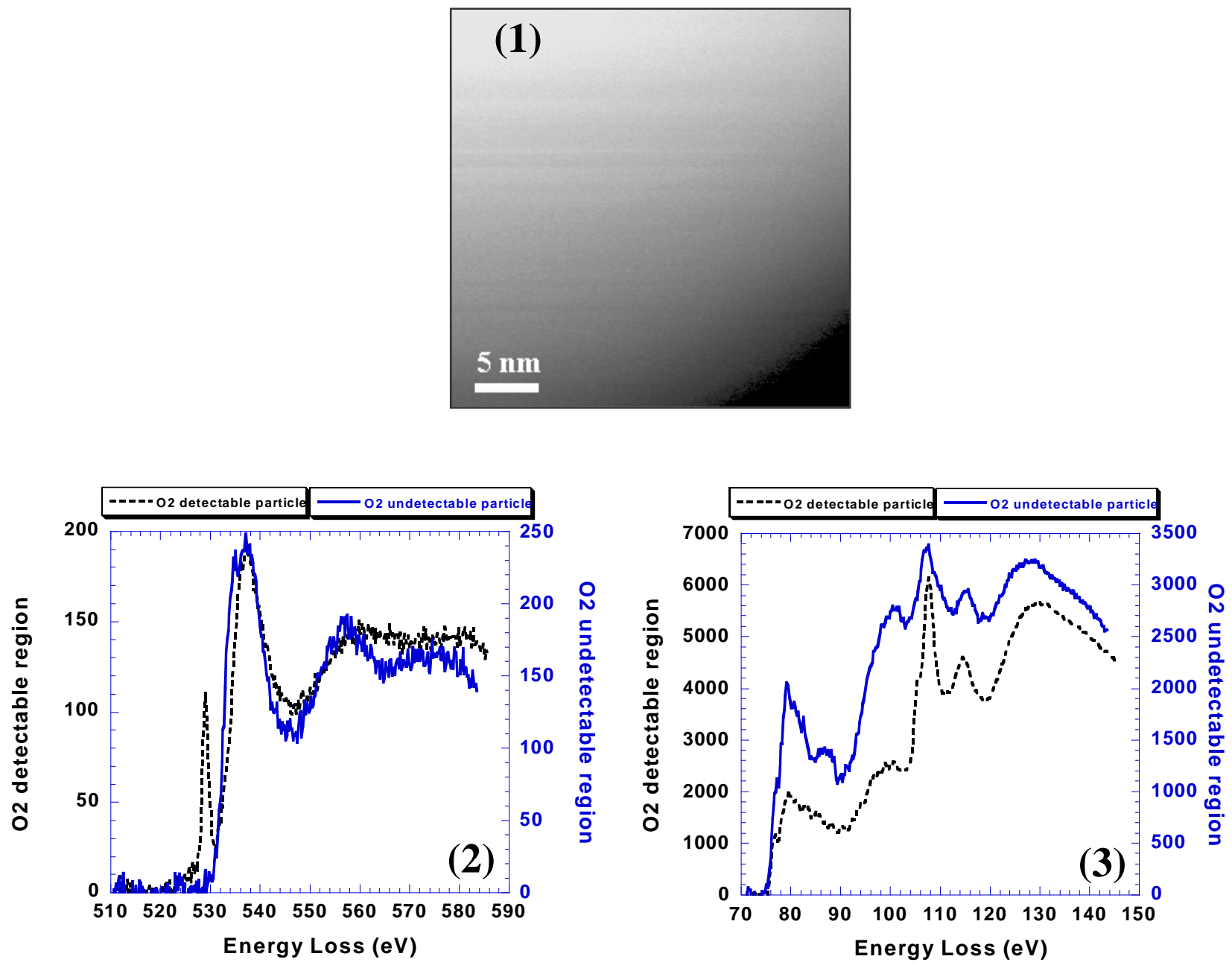

Figure 1. An ADF-STEM image of a calcium aluminosilicate glass particle.

Figure 2. EELS spectra of O K-edges from two different glass particles.

Figure 3. EELS spectra of Al L-edges and Si L-edges from the same two glass particles as in Figure 2. 